\title{
Reseña del libro \\ Bordes, límites y fronteras. \\ Encuentros etnográficos con niños, niñas y adolescentes, editado por Alba Lucy Guerrero, Ángeles Clemente, \\ Diana Milstein \& Maria Dantas-Whitney
}

Review of the book Bordes, límites y fronteras. Encuentros etnográficos

con niños, niñas y adolescentes, edited by Alba Lucy Guerrero, Ángeles

Clemente, Diana Milstein \& Maria Dantas-Whitney

Reseña

Fecha de recepción: 19 de junio de 2019

Fecha de aceptación: 04 de octubre de 2019

Fecha de disponibilidad en línea: abril de 2021

doi: 10.11144/Javeriana.m14.rlbl

CAMilo BÁcares-Jara comalarulfo@hotmail.com

UNIVERSIDAD DEL PAís VASCO, EsPAÑA

(iD) ORCID: https://orcid.org/0000-0002-0508-0869

AnNy Bertoli

anny.bertoli@auckland.ac.nz

Universidad de AuckLand, Nueva Zelanda

(iD) ORCID: https://orcid.org/0000-0003-4195-5869

\section{Palabras clave}

Infancia; investigación; joven; reseña

\section{Keywords}

Childhood; research; youth; book reviews

Para citar este artículo | To cite this article

Bácares-Jara, C. \& Bertoli, A. (2021). Reseña del libro Bordes, límites y fronteras. Encuentros etnográficos con niños, niñas y adolescentes, editado por Alba Lucy Guerrero, Ángeles Clemente, Diana Milstein \& Maria Dantas-Whitney. magis, Revista Internacional de Investigación en Educación, 14, 1-6. doi: 10.11144/ Javeriana.m14.rlbl 
Descripción del artículo | Article description

Reseña del libro de Guerrero, A. L., Clemente, A., Milstein,

D. \& Dantas-Whitney, M. (Eds.). (2017). Bordes, límites

y fronteras. Encuentros etnográficos con niños, niñas y

adolescentes. Editorial Pontificia Universidad Javeriana.

207 pp. ISBN: 978-958-781-156-8.

El libro que reseñamos a continuación no es casual ni está desconectado de un proyecto mayor. A ciencia cierta, es el segundo volumen publicado por los miembros de la Red Internacional de Etnografía con Niños, Niñas, Adolescentes y Jóvenes (RIENN) sobre etnografía colaborativa y niñez, compilado por varias de las mismas autoras y Michael Higgins (Milstein, Clemente, Dantas-Whitney, Guerrero \& Higgins, 2001). Así, lo que se podría nombrar como una antropología de la infancia —en este caso más aplicada al campo educativo - es, quizás, junto a la historia de la infancia y la sociología de la infancia (Castillo, 2015; Gaitán, 2006; Rodríguez-Pascual, 2007; Sosenski, 2016), una de las vertientes disciplinares que más se han interesado por plantear que los niños, las niñas y los adolescentes (NNA) resultan ser productores de conocimiento, quienes, sobre terreno y en su propio contexto, pueden ver los fenómenos de otra manera, sin las ataduras y los códigos de los adultos.

Ciertamente, esta posición se aleja del trabajo etnográfico que en Latinoamérica se ha venido desarrollando en los últimos años, sobre todo en Brasil y Argentina, con los estudios de los dispositivos jurídico-burocráticos de atención a la infancia pobre e infractora (Fonseca \& Cardarello, 2004; Lugones, 2012; Villalta \& Llobet, 2015). A su vez, va más allá del llamado de atención setentero de Mary Ellen Goodman y de Charlotte Hardman (Aristizábal, 2018) de ver a los niños como "informantes antropológicos" o del más reciente que nos heredó el final de la Guerra Fría: que es perentorio y obligatorio que los NNA participen de cualquier proyecto por mandato de la Convención sobre los Derechos del Niño. De este modo, la etnografía colaborativa con NNA le hace el quite a la tendencia políticamente correcta de "darles voz", de incluirlos decorativamente o a modo de un simple requisito metodológico. Su apuesta se adelanta a esto. En esencia, la batalla y el objetivo de la etnografía colaborativa es cocrear conocimiento, romper el monopolio de la palabra y la interpretación de los hechos a cargo de los adultos, y, en una frase, plantearle desafíos metodológicos y epistémicos a la propia antropología y a las formas tradicionales de registrar y pensar etnográficamente las experiencias infanto-adolescentes.

De ahí que este texto tenga como premisa — por eso su título- que los bordes, los límites y las fronteras asociados con la investigación en 
colaboración con los NNA son ante todo una construcción social, cultural e histórica, y que su validez oficial varía y es posible ponerla en entredicho en distintos contextos, infancias y problemáticas. Y es que, dejando de lado las prácticas puntuales de etnografía colaborativa que componen el libro, como, por ejemplo, los primeros cinco capítulos, que tienen lugar en Oaxaca, Oregón, Santiago de Chile, Buenos Aires, La Plata y Jujuy, lo que es realmente prometedor del texto es la reflexión que surge de ellas y las rupturas que provoca el acompañamiento de los NNA en los investigadores, en las categorías utilizadas y en la forma de adelantar la investigación a la hora de hacerse el trabajo de campo.

En esa medida, el libro le permite acercarse al lector a la oportunidad de repensar el concepto de otredad clásico que ha tenido la antropología, es decir, considerar a los NNA como el otro en términos de disminución (Pachón, 2009). Como se sabe, por largo tiempo este otro ha sido un ente generalizado como exótico, remoto, inferior y con una incapacidad de tener pensamiento autónomo e interpretativo (Fabian, 2007). Recién en las últimas décadas estas concepciones han encontrado contestaciones y han empezado a surgir interpretaciones actorales de los más pequeños, incluidos los bebés (Gottlieb, 2004), desde una perspectiva antagónica a la imagen del infans o de alguien reducido a ser gracioso, inocente, tierno y divertido. Justamente, esta nueva alteridad de los NNA que los reconoce como pares interlocutores y que, según Fabian (2006), es el "requisito previo para el tipo de producción de conocimiento que se llama etnografía" (p. 142), es la que se encuentra en cada una de las páginas de este libro. Una otredad que reconoce a los NNA como sujetos capaces de crear conocimiento, de fungir como coinvestigadores, de terminar siendo dialogantes indispensables para entender la realidad estudiada, al igual que de impulsar con su presencia una discusión sobre las categorías preestablecidas del investigador que le impiden acceder a nuevas lecturas de los fenómenos que indaga y le interesan.

Por otra parte, otra ventaja del libro es su postura metodológica y, por ende, cómo se posiciona ante las discusiones al respecto. Partamos de que en la relación etnográfica con la infancia lo habitual ha sido un contínuum de formas de observación caracterizadas por la nula relación del investigador con los NNA, a saber, por la puesta en marcha de un "rol puramente observacional en el que el investigador se sienta fuera de la actividad y no interactúa con los sujetos" (Emond, 2005, p. 125). A ello habría que agregar la disputa contrapuesta a esa versión que tiene lugar entre varios autores que discuten si la observación participante puede ser aplicada en el caso de la investigación con NNA: entre los más clásicos es posible encontrar posiciones que afirman que es "discutible que un investigador adulto asuma 
plenamente el 'papel' de un niño" (James, citado por Emond, 2005) y desde otra comprensión, unas más dadas a invitar a "suspender todas las características típicamente adultas a excepción de la corpórea" (Mandell, 1988, p. 435), sobre todo si la diferencia no es percibida por ellos (Allerton, 2016). De esta manera, en esta última corriente se considera que la relación con los NNA en la etnografía y los procesos participativos que allí se generen es posible solamente si los investigadores adultos logran ocultar su adultez o, en una metáfora, si intentan ponerse un disfraz de infante. Pero lo que es cierto es que en el trabajo que reseñamos ninguna investigación opta por esa infantilización del adulto ni recurre a un antifaz o a un ocultamiento de su condición sociocultural o, si se quiere, de desarrollo, dado que sus planteamientos metodológicos para hacer que "la división entre investigador e investigado se vuelva una línea apenas perceptible" (Guerrero, Clemente, Milstein \& Dantas-Whitney, 2017, p. 17) se fundan en una posición epistémica clara: que los NNA pueden ser algo más que lo que normalmente se ha pensado sobre ellos en clave de control, disminución y paternalismo.

Así pues, el mérito de este libro consiste en reunir un grupo de investigaciones arriesgadas y desatadas de los preformatos hegemónicos sobre la infancia, que en definitiva invitan a quien lea sus páginas a acercarse a las formas, los retos y las oportunidades que ofrece el crear conocimiento con los NNA por medio de la coinvestigación etnográfica. Además, el texto también brinda un recordatorio y una sensación de apoyo una vez se termina de leer: que el conflicto que nos genera el adultocentrismo se puede superar y tratar siempre y cuando entendamos que los bordes, los límites y las fronteras teóricos y metodológicos que le legitiman, inclusive en nuestros proyectos de investigación y en nosotros mismos, se entroncan en representaciones sociales que se perpetúan cada vez que las aceptamos como naturales, obvias y suficientes para la producción de conocimiento.

\section{Los capítulos en detalle}

En el capítulo I, Ángeles Clemente reflexiona acerca de los cambios que se dan en las representaciones sociales de género, edad y clase social, entre otras, en un grupo de estudiantes de la Licenciatura de Inglés en Oaxaca durante una etnografía en colaboración con el otro, en este caso los NNA reclusos o en situación de calle.

El capítulo II, escrito por Maria Dantas-Whitney y Carmen Cáceda, indaga los temas del poder y de la identidad a través de una etnografía en colaboración con un grupo de jóvenes extranjeros que estudian inglés como segunda lengua en la universidad de Oregón, y que experimentan relaciones conflictivas con los "otros" compañeros y profesores. 
En el capítulo III, Andrea Valdivia-Barrios describe la otredad del mundo adulto desde el punto de vista de un grupo de adolescentes que participaron en encuentros de creación audiovisuales en una escuela de Santiago de Chile.

Linda S. Khodr, en el capítulo IV, presenta una investigación en colaboración para entender las acciones políticas de los estudiantes de una escuela secundaria en la provincia de Buenos Aires para defender el nombre de su escuela: "'Che' Guevara".

En el capítulo V, Alejandra Otaso y Santiago Sburlatti ponen en diálogo dos investigaciones en las cuales la colaboración con los NNA permitió resignificar la frontera entre la escuela y sus afueras.

Los últimos dos capítulos toman en consideración los cambios que la colaboración con los NNA produce en los investigadores y en la investigación.

En particular en el capítulo VI, Alba Lucy Guerrero y Diana Milstein relatan acerca de un diálogo espontáneo con Lorena, una niña de 11 años, que se transformó en un encuentro etnográfico cuando Alba Lucy Guerrero reconoció a Lorena como un otro coproductor de conocimiento y puso en discusión su categoría teórica acerca del fenómeno estudiado, el desplazamiento forzado en Colombia.

Finalmente, el último capítulo considera el papel del adulto y de su forma de relacionarse con los coinvestigadores NNA, desde la experiencia de trabajo de campo de Diana Milstein, María Teresa Pujó y Jesús María Jaramillo con un grupo de NNA en un barrio popular de Neuquén, en Argentina.

\section{Sobre los autores}

Camilo Bácares-Jara es doctorando en Educación de la Universidad del País Vasco, España.

Anny Bertoli es doctoranda en Educación, de la Universidad de Auckland, Nueva Zelanda.

\section{Referencias}

Allerton, C. (Ed.). (2016). Children. Ethnographic Encounters. Londres: Bloomsbutry. Aristizábal, D. (2018). Perspectivas y trayectorias teóricas de la antropología de la infancia. Finales del siglo XIX y siglo XX. História Unicap, 5(9), 188-208. doi: 10.25247/hu.2018.v5n9.p187-208

Castillo, P. (2015). Infancia en dictadura. Niñas y niños como testigos: sus producciones como testimonio. Santiago de Chile: Colectivo Infancia y Memoria.

Emond, R. (2005). Ethnographic research methods with children and young people. En S. Greene \& D. Hogan (Eds.), Researching Children's Experience. Approaches and Methods (pp. 123-140). Londres: SAGE. 
Fabian, J. (2006). The Other revisited. Critical afterthoughts. Anthropological Theory, 6(2), 139-152. Recuperado de https://doi.org/10.1177/146349960 6065030

Fabian, J. (2007). Memory against Culture. Londres: Duke University Press.

Fonseca, C. \& Cardarello, A. (2004). Derechos de los más y menos humanos. En S. Tiscornia \& M. V. Pita (Eds.), Derechos humanos, tribunales y policías en Argentina y Brasil. Estudios de antropología jurídica (pp. 7-39). Buenos Aires: Universidad de Buenos Aires.

Gaitán, L. (2006). Sociología de la infancia: nuevas perspectivas. Madrid: Síntesis.

Gottlieb, A. (2004). The Afterlife Is Where we Come from. The Culture of Infancy in West Africa. Chicago: University of Chicago Press.

Guerrero, A. L., Clemente, A., Milstein, D. \& Dantas-Whitney, M. (2017). Bordes, límites y Fronteras. Encuentros etnográficos con niños, niñas y adolescentes. Bogotá: Editorial Pontificia Universidad Javeriana.

Lugones, M. (2012). Obrando en autos, obrando en vidas. Formas y fórmulas de protección judicial en los tribunales prevencionales de menores de Córdoba, Argentina, a comienzos del siglo XXI. Río de Janeiro: E-papers.

Mandell, N. (1988). The least-adult role in studying children. Journal of Contemporary Ethnography, 16(4), 433-467. Recuperado de https://doi.org/10.11 77/0891241688164002

Milstein, D., Clemente, A., Dantas-Whitney, M., Guerrero, A. L. \& Higgins, M. (Eds.). (2001). Encuentros etnográficos con niños, niñas y adolescentes. Entre tiempos y espacios compartidos. Buenos Aires: Miño y Dávila.

Pachón, X. (2009). ¿Dónde están los niños? Rastreando la mirada antropológica sobre la infancia. Maguaré, 23, 433-469. Recuperado de https://revistas. unal.edu.co/index.php/maguare/article/view/15046

Rodríguez-Pascual, I. (2007). Para una sociología de la infancia: aspectos teóricos y metodológicos. Madrid: CIS.

Sosenski, S. (2016). Dar casa a las voces infantiles, reflexiones desde la historia. Revista Latinoamericana de Ciencias Sociales, Niñez y Juventud, 14(1), 4352. Recuperado de http://www.scielo.org.co/pdf/rlcs/v14n1/v14n1a02.pdf

Villalta, C. \& Llobet, V. (2015). Resignificando la protección. Los sistemas de protección de derechos de niños y niñas en Argentina. Revista Latinoamericana de Ciencias Sociales, Niñez y Juventud, 13(1), 167-180. Recuperado de http://biblioteca.clacso.edu.ar/Colombia/alianza-cinde-umz/2015 0512125627/CarlaVillalta.pdf 